\title{
VARIACION RADIAL Y VERTICAL EN LA LONGITUD DE TRAQUEIDAS EN UN ARBOL DE PINO PONDEROSA DE 50 AÑOS (NEUQUÉN, ARGENTINA)
}

\author{
Zingoni, María Inés; Andía, Ismael Ramón; Laffitte, Lorena ${ }^{(1)}$
}

\section{RESUMEN}

El presente trabajo se enmarca en el proyecto de investigación "Características Xilotecnológicas de Pinus ponderosa Dougl. (ex Law.), Cultivado en la Patagonia Andina", a través del cuál se pretende conocer la calidad de la madera que se está formando en las plantaciones de esta especie en la provincia del Neuquén, Argentina, donde se encuentra la mayor superficie forestada.

El objetivo de este trabajo es aportar datos relacionados con la longitud de las traqueidas, los patrones de variación que ocurren en sentido radial y vertical, y la magnitud de dicha variación, por ser este un factor importante en las propiedades de contracción y resistencia, y en el uso de la madera para pulpa y papel.

El estudio se realizó en un individuo seleccionado de un rodal de 50 años, ubicado al SO de la provincia. De secciones transversales del tronco tomadas a diferentes alturas, se obtuvieron tarugos diametrales con orientación norte-sur. Para medir la longitud de las traqueidas, se maceraron astillas del leño temprano de los anillos pares y se utilizaron los datos obtenidos en dirección médula-corteza para delimitar la zona de madera juvenil y madura por métodos de regresión.

En sentido radial, la longitud de las traqueidas aumenta hasta un determinado número de anillo (zona de madera juvenil) y luego se estabiliza (zona de madera madura). El porcentaje promedio de variación de la zona juvenil fue del $160 \%$, y de la zona madura del 12\%. Se encontró una fuerte relación lineal, entre la pendiente de la recta de regresión de la zona juvenil con la edad de formación de las iniciales cambiales, lo que explica que el cambium formado cuando el árbol es más viejo comienza a formar madera madura en anillos mas cercanos a la médula.

En sentido vertical la tendencia es a aumentar hasta los $9.3 \mathrm{~m}$, con porcentajes de variación del $71 \%$ en la zona juvenil y del $27 \%$ en la zona madura, luego disminuye a mayores alturas del árbol.

El valor promedio de longitud de traqueida es de $2.264 \mu \mathrm{m}$ y $3.389 \mu \mathrm{m}$ para la zona juvenil y madura, respectivamente.

Palabras clave: Variabilidad de la madera, calidad de la madera, coníferas exóticas.

(1) UNIVERSIDAD NACIONAL DEL COMAHUE. NEUQUEN - ARGENTINA. acad_ausma@yahoo.com 


\section{SUMMARY}

This paper is part of the research project "Xylotechnological Characteristics of Cultivated Ponderosa Pine in Andean Patagonia". The Project pretends to study the wood quality that is being formed in plantations of this species in the province of Neuquén, where can be found the main cultivated area with the species..

The aim of this paper is to contribute with data related to tracheids length, vertical and radial variation patterns and magnitude of such variation. This anatomic characteristic influences on the shrinkage and resistance properties and on the use of wood for paper.

The study was carried out in a specimen selected from a 50 years old stand located in the south west of the province. Strips in a north- south direction were cut from crosssections at various levels of the tree trunk. To measure tracheids length, early wood was macerated in the even-numbered growth rings and the data obtained in pith-bark direction were used to determine the boundary between juvenile and mature wood by regression methods.

Radially, the tracheids length increases up to a specific number of ring (juvenile wood zone) and then, it remains steady (mature wood zone). The average percentage of juvenile zone variation was of $160 \%$ and in the mature zone of $12 \%$. A strong lineal relation was found between the slope of the regression line of the juvenile wood and the formation age of the cambial initials. This means that the cambium formed when the tree is older forms mature wood in the growth rings which are nearer the pith.

Vertically, the tendency is to increase up to $9.3 \mathrm{~m}$, with variation percentages of $71 \%$ in the juvenile zone and $27 \%$ in the mature zone. It diminishes at higher tree levels.

The average value of tracheid length is $2264 \mu \mathrm{m}$ and $3389 \mu \mathrm{m}$ for juvenile and mature zones, respectively.

Key Words: Wood variability, wood quality, exotic conifers 


\section{INTRODUCCIÓN}

La demanda creciente de madera para diferentes usos ha llevado a implantar especies exóticas de rápido crecimiento en diferentes regiones del país.

En la región andino-patagónica el objetivo de estas plantaciones es obtener madera para su uso como material sólido, contribuyendo además a disminuir la presión sobre el bosque nativo, cuyo producto ha sido utilizado de manera indiscriminada para diferentes aplicaciones.

La especie más representativa que ha sido plantada con estos fines a partir del año 1978, es Pinus ponderosa (pino ponderosa). Definir las propiedades que presenta la madera de esta especie resulta dificultoso por la gran variación que existe entre los rodales respecto del sitio, edad, manejo silvicultural y origen de las semillas. Se han realizado algunos trabajos enfocados al análisis de algunas de sus propiedades (Bianchet, 1979; Jovanovsky et al., 2002; Lomagno, 2005; Zingoni et al , 2005; Andía et al., 2007) los que son aún insuficientes.

En la actualidad es necesario incorporar el concepto de calidad de madera en plantaciones con fines productivos y realizar investigaciones en este sentido, ya que las que se refieren a cantidad de madera son las que más se orientaron en el estudio particular de pino ponderosa creciendo en la patagonia andina.

Es conocido que la madera varía dentro de un árbol, entre árboles de un mismo rodal y entre sitios, especialmente en coníferas, muchos trabajos lo confirman, por lo que es necesario cuantificar tal variación para predecir posibles usos y planificar la plantación y posterior manejo en función del producto que se desea obtener.

Una de las características anatómicas que afecta algunas propiedades de la madera, es la longitud de las traqueidas, y su variación en el fuste, tanto en sentido radial como vertical, sigue patrones semejantes en diferentes especies, como Pinus taeda (Jackson, 1959; Zobel y van Buijtenen, 1989), Larix laricina (Yang et al., 1986), Picea mariana y P. glauca (Yang, 1994), Pinus radiata (Dadswell, 1958), Pinus caribaea (Schmidt y Smith, 1961; Andrews y Hughes, 1973), Pinus densiflora (Sakai y Vegaki, 1962), Sequia sempervirens (Orell, 2004,). Lo que es diferente para cada especie es la magnitud de la variación experimentada y la manera en que ésta es afectada por factores internos, como la genética y la edad, y otros, como el sitio y las prácticas silviculturales.

\section{OBJETIVOS}

El objetivo general de este estudio es aportar datos para la caracterización xilotecnológica de pino ponderosa, por ser la especie de conífera más representativa de las plantaciones de la patagonia andina.

Los objetivos específicos son determinar la longitud de las traqueidas y analizar su variación, de acuerdo a su ubicación en altura y diámetro en el fuste, en un árbol maduro, 
dado que es un factor importante en la calidad de la madera para pulpa y papel y en las propiedades de contracción y resistencia.

\section{MATERIAL Y MÉTODO}

El lugar de estudio fue el Arboretum Lote 69, ubicado en el Departamento Lacar, provincia del Neuquén ( $40^{\circ} 10^{\prime} \mathrm{LS}$ y $71^{\circ} 20^{\prime} \mathrm{LW}$ ), a $1000 \mathrm{msnm}$, con temperatura media anual de $9^{\circ} \mathrm{C}$ y precipitación media anual de $1300 \mathrm{~mm}$. El suelo corresponde al orden andisoles, cuyo material de origen es de cenizas volcánicas, bajo un régimen de humedad permanente. El suelo es profundo, con buena permeabilidad y alta retención hídrica.

Si bien las plantaciones de pino ponderosa se iniciaron en la región de manera sostenida, hace aproximadamente 30 años, este estudio se realizó en un rodal plantado con fines experimentales en el año 1952, considerando que en función de los objetivos planteados, el árbol muestra debía superar lo máximo posible la edad establecida para la corta final de esta especie, que es entre los 35 y 40 años.

Se seleccionó un árbol de posición sociológica dominante, de buen fuste y estado sanitario (Figura $\mathrm{N}^{\circ} 1$ ), que fue apeado en diciembre de 2002. Se cortaron discos a distintas alturas, desde los 0,30 m a intervalos de $3,0 \mathrm{~m}$, y se obtuvieron 10 tarugos diametrales orientados norte - sur (Figura $N^{\circ} 2$ ).

De cada tarugo se tomaron muestras de astillas del leño temprano de todos los anillos pares, las que fueron maceradas por el método de Franklin (1945). La longitud de traqueida establecida para cada anillo, fue el promedio resultante de la medición de una muestra de 30, cuyo tamaño fue determinado por el método sugerido por Yang y Pulkki (2002). Para las mediciones se utilizó microscopio binocular con ocular micrométrico.

El límite entre madera juvenil y madura, de la orientación norte y sur a las distintas alturas del tronco fue determinado utilizando el método de regresión de los tres pasos propuesto por Yang et al. (1986).

Los tres últimos tarugos (8-10), correspondientes a las partes más altas del árbol, no fueron incluidos en el análisis de las relaciones por considerar que el número de anillos de crecimiento no fue suficiente para demarcar con la mayor precisión posible el límite entre madera juvenil y madura.

La edad de formación de las iniciales cambiales para los diferentes niveles se determinó con la siguiente fórmula:

$$
E F I C=A_{(0,3 m)}-A_{(n)}+1
$$

Donde:

$$
\begin{aligned}
& A_{(0,3 m)}: \text { Número de anillos a los } 0,3 \mathrm{~m} \\
& A_{(n)}: \text { Número de anillos de un determinado nivel }
\end{aligned}
$$




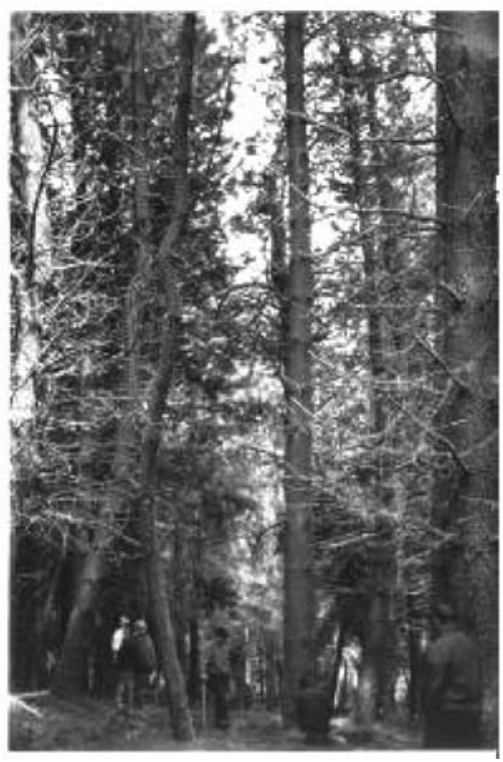

Figura $\mathrm{N}^{\circ} 1$

ARBOL DOMINANTE DE PINUS PONDEROSA

DE 50 AÑOS. -RODAL ARBORETUM LOTE 69. SO DE NEUQUÉN. ARGENTINA

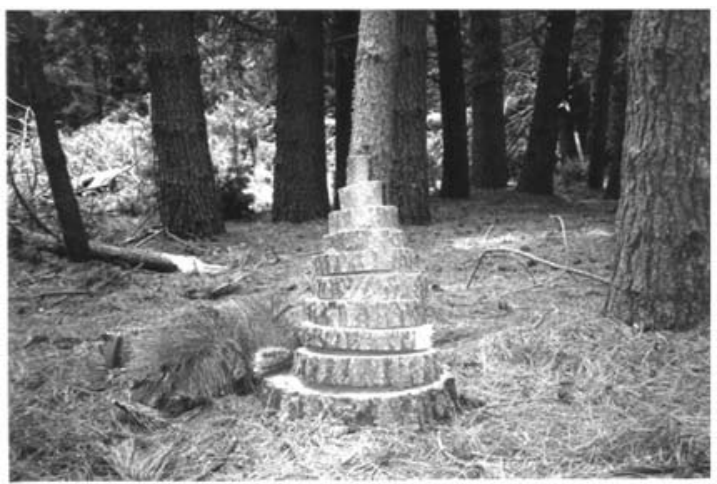

Figura $\mathrm{N}^{\circ} 2$

DISCOS OBTENIDOS CADA $3 \mathrm{~m}$, A PARTIR DE 0,3 m 


\section{RESULTADOS}

En la Figura $N^{\circ} 3$ se muestra la variación radial de la longitud de las traqueidas a diferentes alturas y las rectas de regresión para las zonas de madera juvenil y madera madura, cuya intersección fue considerada como el límite entre ambas.

Los datos del árbol muestra y la extensión expresada en número de anillos para madera juvenil y madura, se presentan en el Cuadro $\mathrm{N}^{\circ} 1$.

\section{Cuadro $\mathrm{N}^{\circ} 1$.}

\section{EXTENSIÓN DE MADERA JUVENIL Y MADERA MADURA A DIFERENTES}

ALTURAS Y EDAD DEL NIVEL

\begin{tabular}{|ccccccc|}
\hline $\begin{array}{c}\text { Nivel } \\
\left(\mathbf{N}^{\circ}\right)\end{array}$ & $\begin{array}{c}\text { Altura } \\
(\mathbf{m})\end{array}$ & $\begin{array}{c}\text { Edad } \\
(\text { años })\end{array}$ & \multicolumn{2}{c}{$\begin{array}{c}\text { Madera Juvenil } \\
\left(\mathbf{N}^{\circ} \text { de anillos }\right)\end{array}$} & \multicolumn{2}{c|}{$\begin{array}{c}\text { Madera Madura } \\
\left(\mathbf{N}^{\circ} \text { de anillos) }\right.\end{array}$} \\
\hline 1 & 0,3 & 49 & 18 & 20 & Norte & Sur \\
\hline 2 & 3,3 & 43 & 15 & 18 & 28 & 29 \\
3 & 6,3 & 40 & 17 & 18 & 23 & 22 \\
4 & 9,3 & 35 & 13 & 13 & 22 & 22 \\
5 & 12,3 & 31 & 10 & 9 & 21 & 22 \\
6 & 15,3 & 26 & 11 & 10 & 15 & 16 \\
7 & 18,3 & 21 & 10 & 9 & 11 & 12 \\
\hline
\end{tabular}

La variación de la longitud de las traqueidas a distintas alturas en la zona de madera juvenil tomando los anillos 2, 4 y 6 contados a partir de la médula y en la zona de madera madura tomando los anillos 2, 4 y 6 contados a partir de la corteza, se muestran en la Figura $\mathrm{N}^{\circ} 4$. 


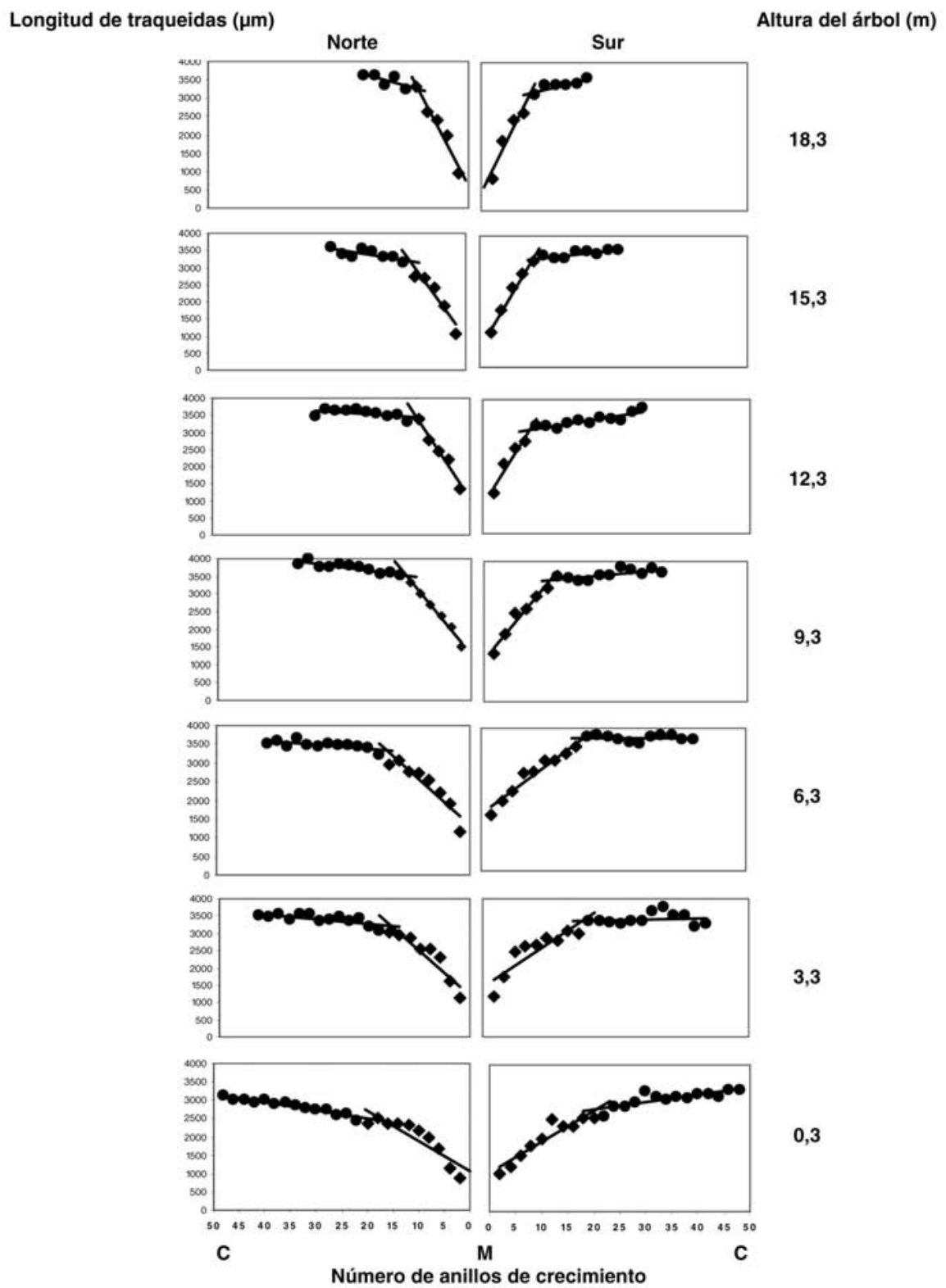

Figura $\mathrm{N}^{\circ} 3$

VARIACIÓN RADIAL DE LA LONGITUD DE TRAQUEIDAS 


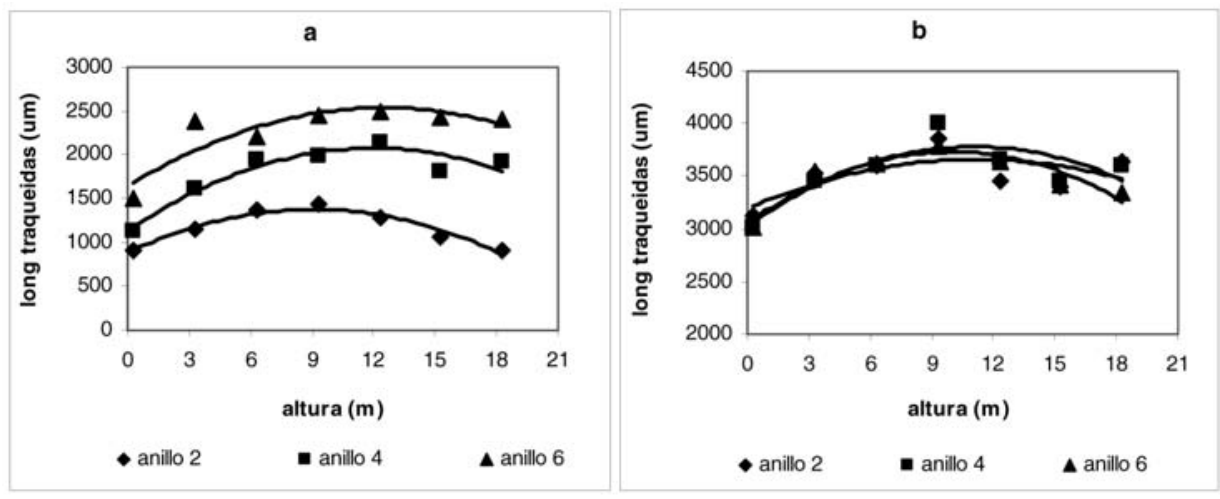

Figura $N^{\circ} 4$

VARIACIÓN DE LA LONGITUD DE LAS TRAQUEIDAS CON LA ALTURA DEL TRONCO.

a: anillos 2, 4 y 6 desde la médula a la corteza (madera juvenil);

b: anillos 2, 4 y 6 desde la corteza a la médula (madera madura)

Los valores de "a" (ordenada al origen) y de "b" (pendiente), de las rectas de regresión de la zona juvenil, se relacionaron con la edad de formación de las iniciales cambiales. Los valores de "a" fueron considerados como la longitud de las iniciales del cambium. (Cuadro N²; Figura $\mathrm{N}^{\circ} 5$ y 6).

\section{Cuadro $\mathrm{N}^{\circ} 2$.}

VALORES DE LA ORDENADA AL ORIGEN (a) Y DE LA PENDIENTE (b) DE LAS RECTAS DE REGRESION DE LA ZONA JUVENIL A DISTINTAS ALTURAS Y EDAD DE FORMACION DE LA INICIAL CAMBIAL (fic)

\begin{tabular}{|cccccc|}
\hline Altura & Edad fic & \multicolumn{2}{c}{ a -longitud inicial cambial $(\mu \mathrm{m})$} & \multicolumn{2}{c|}{ b -pendiente } \\
$(\mathbf{m})$ & (años) & Norte & Sur & Norte & Sur \\
\hline 0,3 & 1 & 1064 & 899 & 83 & 76 \\
3,3 & 7 & 1210 & 1471 & 129 & 102 \\
6,3 & 10 & 1312 & 1561 & 122 & 113 \\
9,3 & 15 & 1259 & 1160 & 177 & 184 \\
12,3 & 19 & 1036 & 879 & 232 & 253 \\
15,3 & 24 & 912 & 623 & 208 & 273 \\
18,3 & 29 & 633 & 511 & 270 & 291 \\
\hline
\end{tabular}




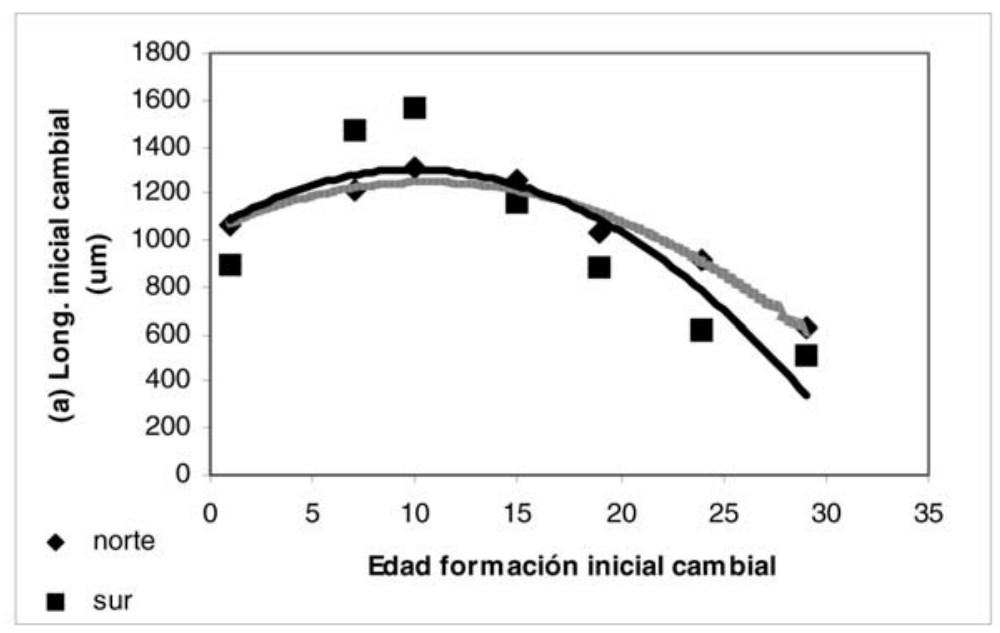

Figura $\mathrm{N}^{\circ} 5$

RELACIÓN ENTRE LONGITUD DE LA INICIAL CAMBIAL (a) Y EDAD DE FORMACIÓN DE LA INICIAL CAMBIAL.

Norte : $y=-1,9013 X^{2}+40,387 x+1039,8 ;$ Sur : $y=-2,666 X^{2}+53,223 x+1036,5$

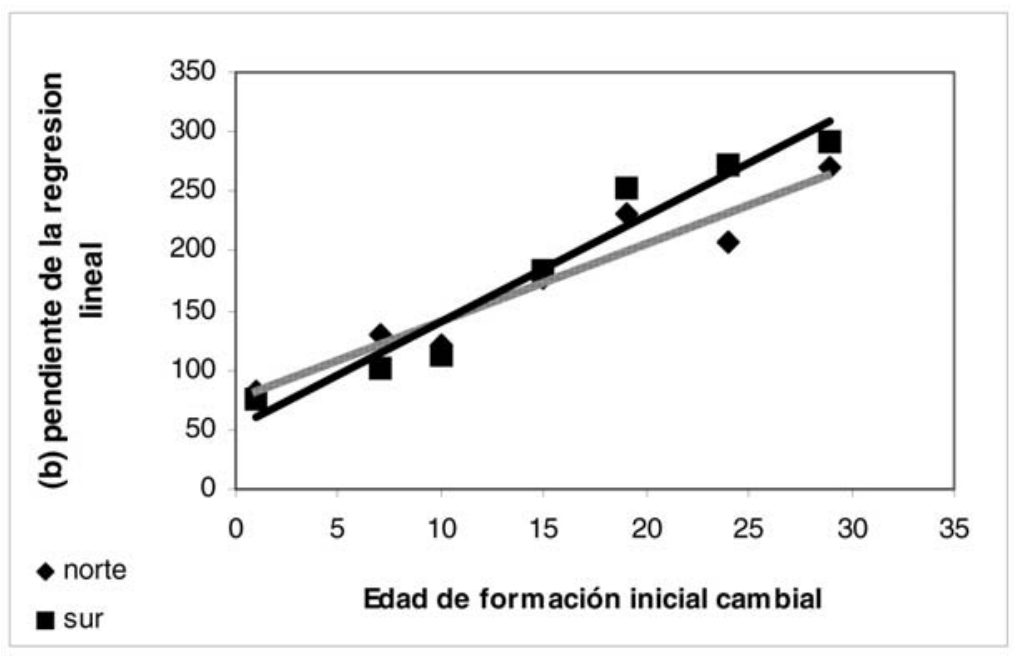

Figura $\mathrm{N}^{\circ} 6$

RELACIÓN ENTRE LA PENDIENTE DE LA REGRESIÓN LINEAL (B)

Y LA EDAD DE FORMACIÓN DE LA INICIAL CAMBIAL (fic)

Norte: $y=6,5329 x+76,435 ;$ Sur: $y=8,8201 x+52,27$ 


\section{Cuadro $\mathrm{N}^{\circ} 3$ \\ LONGITUD PROMEDIO DE TRAQUEIDAS $(\mu \mathrm{m})$ Y DESVÍO ESTANDAR DE MADERA JUVENIL (MJ) Y MADERA MADURA (MM) A DISTINTAS ALTURAS EN EL TRONCO}

\begin{tabular}{|cccccccccccc|}
\hline $\begin{array}{c}\text { Altura } \\
(\mathbf{m})\end{array}$ & \multicolumn{4}{c}{ Norte } & \multicolumn{3}{c}{ MJ } & \multicolumn{2}{c}{ MM } & \multicolumn{3}{c}{ MJ } & MM & MJ & MM \\
\hline 0,3 & 1932 & 575 & 2801 & 224 & 1735 & 487 & 2694 & 181 & 1833 & 2747 \\
3,3 & 2277 & 672 & 3377 & 172 & 2490 & 634 & 3409 & 163 & 2384 & 3393 \\
6,3 & 2413 & 644 & 3471 & 108 & 2693 & 637 & 3728 & 80 & 2553 & 3599 \\
9,3 & 2497 & 665 & 3740 & 140 & 2446 & 705 & 3625 & 139 & 2471 & 3682 \\
12,3 & 2427 & 750 & 3545 & 117 & 2146 & 683 & 3376 & 180 & 2286 & 3460 \\
15,3 & 2160 & 700 & 3344 & 139 & 2263 & 873 & 3465 & 109 & 2212 & 3405 \\
18,3 & 2250 & 878 & 3482 & 179 & 1966 & 787 & 3388 & 146 & 2108 & 3435 \\
Promedio & 2279 & 698 & 3394 & 154 & 2248 & 686 & 3383 & 143 & 2264 & 3389 \\
\hline
\end{tabular}

Los valores promedio de longitud de traqueidas para toda la madera juvenil y madura, orientación norte y sur se resumen en el Cuadro $\mathrm{N}^{\circ} 3$.

Del análisis estadístico surge que no hay diferencias significativas a un 95\% entre las medias de las dos orientaciones tanto dentro de la zona juvenil, como en la zona madura, mientras que las medias entre la zona juvenil y madura son significativamente diferentes unas de otras.

\section{DISCUSIÓN Y CONCLUSIONES}

Desde la médula hacia los últimos anillos cercanos a la corteza y considerando diferentes alturas del tronco y la orientación norte y sur, la longitud de las traqueidas aumenta hasta un determinado número de anillo a partir del cual los valores se presentan más o menos constantes. Para el nivel $1(0,30 \mathrm{~m})$, la longitud de las traqueidas parece estabilizarse en los anillos 18 y 20 para la orientación norte y sur, respectivamente. En alturas superiores ocurre en anillos más cercanos a la médula.

Este patrón es similar al descripto para otras especies de coníferas en las que la longitud de las traqueidas se estabiliza en los anillos 12-15 para Pinus radiata (Daswell, 1958), 19 para Pinus caribaea (Schmidt y Smith, 1961), 14-16 para Picea mariana (Yang, 1994), 12-16 para Picea glauca (Yang, 1994), 13 para Pinus banksiana (Bodie, 1988), 44 para Larix laricina (Yang et al., 1986), 24 para Cryptomeria japonica (Yang y Chen, 1994), 35 para Pinus sylvestris (Panshin y Zeeuw, 1980), 25 para Cupressus lusitanica y Cupressus macrocarpa (Panshin y Zeeuw, 1980), y 18 para Pinus taeda (Larson et al., 2001). Cuanto más longeva sea la especie tardará mayor cantidad de años en alcanzar los valores más altos de longitud (Panshin y Zeeuw, 1980).

Si bien los patrones son similares, la magnitud de la variación del largo de traqueida experimentada en sentido radial es diferente para cada especie. 
En este estudio para pino ponderosa, el porcentaje de variación de la longitud de las traqueidas entre el primer anillo que rodea a la médula y el anillo más externo, en las diferentes alturas del tronco, es superior al 163\%, alcanzando el valor más alto a los 18,30 $\mathrm{m}$, que fue de 297\%; lo que muestra que el cambio fue de 1,5 a 3 veces mayor. Variaciones similares se encontraron en Pinus radiata (Dadswell 1958); Pinus caribaea (Plumptre 1983) y Pinus taeda (Zobel y van Buijtenen, 1989). El porcentaje de variación teniendo en consideración la zona juvenil por un lado y la zona madura por otro, presentó valores promedio de $160 \%$ y $12 \%$, respectivamente.

La variación en el largo de traqueida con la altura del árbol, se analizó en los anillos 2, 4 y 6 contados a partir de la médula, los que presentan una tendencia similar. Aumenta hasta los 12,3 m (anillo 4 y 6 ) y hasta los 9,3 m (anillo 2) y luego disminuye (Figura $\mathrm{N}^{\circ}$ 4a). Los valores se presentan progresivamente más elevados desde el anillo 2 hasta el 6, correspondiéndose con el aumento que experimenta el largo en sentido radial en la zona de madera juvenil.

En la zona de madera madura donde la longitud se estabiliza, fueron analizados los anillos 2, 4 y 6 contados a partir de la corteza. En éstos se observa un aumento en la longitud de las traqueidas hasta los 9,3 m y una disminución hacia mayores alturas (Figura $\left.N^{\circ} 4 b\right)$.

El porcentaje de variación es menor en la zona de madera madura, donde alcanza un aumento del $27 \%$ respecto de la zona de madera juvenil que representa el $71 \%$, para luego ir disminuyendo hacia las partes más altas del árbol. Un patrón similar al descripto encontró Jackson (1959), analizando Pinus taeda. De cualquier manera, la mayor variabilidad se presenta en sentido médula - corteza.

Estimada a partir de la intercepción (a) de la ecuación lineal para longitud de traqueida de la zona juvenil al nivel del tronco correspondiente, la longitud de las iniciales cambiales muestra una relación curvilínea con la edad de su formación, (Orientación Norte, $R^{2}=0,9597$ y Orientación Sur, $R^{2}=0,7499$ ) aumentando hasta los 10 años y luego comienza a disminuir a medida que aumenta la edad de formación (Figura $N^{\circ} 5 a$ ).

La pendiente (b) de la recta de regresión de la longitud de traqueida de la madera juvenil muestra una fuerte relación positiva con la edad de formación de las iniciales cambiales (Orientación Norte, $\mathrm{R}^{2}=0,922$ y Orientación Sur, $\left.\mathrm{R}^{2}=0,9455\right)$, (Figura $\mathrm{N}^{\circ} 6$ ). Esto indica que el cambium formado recientemente, cuando el árbol es más viejo, forma madera madura en un período más corto que el cambium desarrollado en etapas más tempranas de la vida del árbol.

Este patrón se encontró en otras coníferas y en dicotiledóneas y apoya el concepto que relaciona la transición de madera juvenil a madera madura con el proceso de maduración de las iniciales del cambium (Yang et al., 1986).

Los patrones de variación en sentido radial y vertical analizados en este estudio de caso muestran la influencia de la edad en la longitud de las traqueidas. La edad de 
transición de madera juvenil a madura, determinada utilizando la longitud de las traqueidas resultó un criterio útil y coincide con lo citado por Loo et al. (1985), analizando la variación radial de la densidad de pino ponderosa

Los valores promedio de la longitud de las traqueidas hallados en la madera juvenil $(2264 \mu \mathrm{m})$ y madura $(3389 \mu \mathrm{m})$ son similares a los citados por Lomagno (1998) para plantas de 18 años $(1.300 \mu \mathrm{m})$ en los primeros anillos a $2.200 \mu \mathrm{m}$ en la zona externa) dimensiones que se corresponden con la edad de las plantas las que se consideran formadas en su totalidad por madera juvenil. Sucate et al.(1978) en plantaciones de esta especie provenientes de Río Negro midieron en árboles de 24 años, longitudes de $3.290 \mu \mathrm{m}$, valores mas altos que podrían deberse a la presencia de madera madura. De cualquier manera, pino ponderosa presenta traqueidas más cortas que otras especies de pino de interés comercial que se cultivan en la zona del noreste de la Argentina.

Los datos obtenidos en este trabajo, respecto de la variación radial y vertical de la longitud de las traqueidas, y su utilización para delimitar la madera juvenil de la madera madura en pino poderosa, son los primeros reportes relacionados con estos aspectos en plantaciones de Argentina.

La longitud de las traqueidas es considerado un carácter altamente heredable (Larson et al., 2001) y factores como las características del sitio y las técnicas silviculturales pueden tener efectos diferentes según la especie y aún entre individuos de la misma especie; así lo demuestra la extensa bibliografía al respecto.

Por esto es necesario promover estudios con el objetivo de analizar la influencia de factores intrínsecos y extrínsecos sobre el largo de las traqueidas y extensión de madera juvenil en pino ponderosa cultivado en la región andino-patagónica, considerando que estos son dos parámetros importantes de calidad de madera.

\section{RECONOCIMIENTOS}

El presente trabajo se realizó en el marco del Proyecto de Investigación "Características xilotecnológicas de Pinus ponderosa (Dougl. ex Laws) cultivado en la patagonia andina", aprobado y subsidiado por la Secretaría de Investigación de la Universidad Nacional del Comahue - Argentina.

\section{REFERENCIAS}

Andía, I. A.; Zingoni, M. I.; Jara, O.y Mantilaro, N., 2007. Contracciones Transversales y Longitudinal en Madera Juvenil de Pino Ponderosa Proveniente de Plantaciones del Norte de Neuquén. Actas del III Congreso Iberoamericano de Productos Forestales. ISSN 1851-0965. $7 \mathrm{pp}$

Andrews, I. A. y Hughes, J. F., 1973. Variation in Wood Properties in 12-year-old Trees of Pinus caribaea. Hondurensis in Trinidad - a summary report. Trop. Prov. Prog. Res. Int . Coop. Nairobi, Kenya, 532-535 
Biachet, J. M., 1979. Ensayos Físico-Mecánicos en Madera de Coníferas. Instituto Forestal Nacional , Folleto Técnico N³8, 23 pp

Bodie, B. F., 1988. Wood Properties of Jack Pine from a Northwestern Ontario Plantation. B.S. Thesis School For. Lakehead Univ. Thunder Bay. Ontario. Canadá

Dadswell, H. E., 1958. Wood Structure Variations Occurring During Tree Growth and their Influence on Properties. J. Inst. Wood Sci 1: 11-33

Jackson, L. W., 1959. Loblolly Pine Tracheid Length in Relation to Position in Tree. J. For. 57: $366-367$

Jovanovski, A.; Jaramillo, M.; Loguercio, G. y Antequera, S., 2002. Densidad de la Madera de Pinus ponderosa (Dougl. Ex Laws) en Tres Localidades de Argentina. Bosque 23 (2): 99-104

Larson, P. R, 1969. Wood Formation and the Concept to Wood Quality. Yale Univ. School of Forestry Bulletin 74, $54 \mathrm{pp}$

Larson, P. R.; Kretschmann, D.; Clark III, A. e Isebrands, J. G., 2001. Formation and Properties of Juvenile Wood in Southern Pines. A synopsis. USDA Forest Products Laboratory, General Thecnical Report, 1-49.

Lomagno, J. L., 1998. La Utilización de Raleos de Pino ponderosa. CIEFAP Pub. Tec. $\mathrm{N}^{\circ} 28,19 \mathrm{pp}$

Lomagno, J. L., 2005. Contracción Longitudinal de la Madera de Pino Ponderosa. Variación entre Rodales. Actas del Tercer Congreso Forestal Argentino y Latinoamericano. ISSN 0329-1103. 9 pp

Loo, J. A.; Tauer, C.G. y McNew, R. W., 1985. Genetic Variation in the Time of Transition from Juvenile to Mature Wood in Loblolly Pine (Pinus taeda). Silvae Genet. 34(1): 14-19

Orell, M., 2004. Estudio del Largo de Traqueidas en Sequoia sempervirens D. Don (Endl) Crecida en Chile, con Respecto a su Ubicación en el Árbol. Tesis Ing. Forestal, Univ. Chile

Panshin, A. J. y Zeeuw, C., 1980. Textbook of Wood Technology. $4^{\text {th }}$ ed. Mc Graw-Hill. N.Y. 643 pp.

Plumptre , R. A., 1983. Pinus caribaea Vol II Wood Properties. Trop. For. Pap. 17 For. Inst. Oxford Univ. $145 \mathrm{pp}$

Sakai, H. y Uegaki, T., 1962. On the Structure of the Annual Ring of Japanese Red Pine (Pinus densiflora). Variation of Annual Ring Width, Specific Gravity and Tracheid Length in Young Tree Stems. Tottori Soc Agr Sci 14:113-119 
Schmidt, J. D. y Smith, W. J., 1961. Wood Quality Evaluation and Improvement in Pinus caribaea. Queenst For Serv Res Note 15. p 59

Sucate, S.; Garona, M. y Merlo, J., 1978. Evaluación de Cuatro Especies de Pinos del Sur para Uso Papelero. Pub. CICELPA N² 29, 25 pp

Yang, K. C.; Benson C. A. y Wong, J. K., 1986. Distribution of Juvenile Wood in Two Stems of Larix laricina. Can J. For. Res. 16: 1041-1049

Yang, K. C. y Chen Y. S., 1994. Formation and Vertical Distribution of Juvenile and Mature Wood in a Single Stem of Cryptomeria japonica. Can J. For. Res. 24: 969-975

Yang, K. C., 1994. Impact of Spacing on Width and Basal Area of Juvenile and Mature Wood in Picea mariana and Picea glauca. Wood and Fiber Sci 26(4): 479-488

Yang, K. C. y Pulkki, R. E., 2002. Sample Size Determination and Probability Level Estimation. Taiwan J. For. Sci. 17 (2) 135-141

Zingoni, M. I.; Andía, I. R.; Guerra, P. E. y Mele, U .E., 2005. La Madera Juvenil: un Aspecto de Importancia a Considerar en Plantaciones de Pinus ponderosa. Actas del Tercer Congreso Forestal Argentino y Latinoamericano. ISSN 0329-1103. 9 pp

Zobel, B. J.; van Buijtenen, J. P., 1989. Wood Variation. Its causes and Control. SpringerVerlang. 363 pp. 